\title{
Pharmacology and phytochemistry of the Nitraria genus (Review)
}

\author{
QIAOHUI DU ${ }^{1,2}$, HAILIANG XIN ${ }^{2}$ and CHENG PENG ${ }^{1}$ \\ ${ }^{1}$ Key Laboratory of Standardization of Chinese Herbal Medicines of the Ministry of Education, Pharmacy College, \\ Chengdu University of Traditional Chinese Medicine, Chengdu, Sichuan 610072; ${ }^{2}$ Department of Traditional \\ Chinese Medicine, Changhai Hospital, Second Military Medical University, Shanghai 200433, P.R. China
}

Received December 17, 2013; Accepted June 9, 2014

DOI: $10.3892 / \mathrm{mmr} .2014 .2677$

\begin{abstract}
Plants from the Nitraria genus, members of the Zygophyllaceae family, grow naturally in Europe, Africa, Australia and the central Asian desert. Previous pharmacological research has provided evidence that members of the Nitraria genus have numerous beneficial effects. In the present review, the pharmacological and phytochemical studies of Nitraria were presented and assessed. The review was written using information published between 1968 and 2013 from a number of reliable sources, including ScienceDirect, Springer, PubMed, EMBASE and CNKI. Numerous compounds, such as alkaloids and flavonoids have been isolated from the plants of this genus in the past, and multiple members of these constituents have been demonstrated to exert antitumor or anti-oxidative activities. The extracts of plants of the Nitraria genus possess antitumor, antiproliferative, anti-oxidative, antifatigue, anti-mutagenic, antimicrobial, hypotensive, hepatoprotective, lipid-lowering and hypoglycemic effects. However, the possible active components in the fraction and the molecular mechanisms require further investigation prior to their use in clinical practice.
\end{abstract}

\section{Contents}

1. Introduction

2. Phytochemical studies

3. Pharmacological effects

4. Conclusion

Correspondence to: Dr Hailiang Xin, Department of Traditional Chinese Medicine, Changhai Hospital, Second Military Medical University, 168 Changhai Road, Shanghai 200433, P.R. China E-mail: hailiangxin@163.com

Dr Cheng Peng, Key Laboratory of Standardization of Chinese Herbal Medicines of Ministry of Education, Pharmacy College, Chengdu University of Traditional Chinese Medicine, 37 Shi'erqiao Road, Chengdu, Sichuan 610072, P.R. China

E-mail: pengchengchengdu@126.com

Key words: plant medicine, pharmacological action, phytochemistry, Nitraria, Zygophyllaceae

\section{Introduction}

The Nitraria genus, a member of the family Zygophyllaceae, is one of the dominant species in the Mediterranean and central Asian deserts. The English name Nitre-bush is from the Latin word saltpetre, referring to the fact that it can thrive in saline soil. It serves an key ecological role due to its superior tolerance to severe drought and high salinity. Nitraria are shrubs 25-200-cm tall, with spiny branches at the apex and simple serrated leaves. The flowers are yellowish-gray or white, 2-4 cm pubescent, with five petals and five sepals, while the fruit is a fleshy drupe. The Nitraria genus consists of 13 species across the world, which are mainly distributed in Asia, Europe, Africa and Australia (Table I).

A growing body of evidence now demonstrates that Nitraria extract has numerous biomedical properties, including antifatigue, antitumor, anti-oxidative and antimutagenic activities. In addition, the fruit of Nitraria sibirica is extensively used to treat hypertension, menstrual disorders and gastroenteritis in folklore medicines of Northwest China $(1,2)$. The leaves of Nitraria retusa also serve as supplement tea and are used as a poultice in Africa $(3,4)$.

To provide further support and evidence for the clinical use of this genus, a systematic review of the modern phytochemical and pharmacological properties of Nitraria was performed. The available information on the pharmacology and phytochemistry of the Nitraria genus was collected via libraries and electronic searches using PubMed (http://www.ncbi.nim. nih.gov/pubmed), ScienceDirect (http://sciencedirect.com), Springer (http://springer.com), EMBASE (http://elsevier.com/ online-tools/embase) and CNKI (http://www.cnki.net).

\section{Phytochemical studies}

Currently, the following six species of the Nitraria genus have been observed for their phytochemical properties: $N$. komarovii, N. schoberi, $N$. sibirica, N. tangutorum, $N$. retusa and $N$. billardieri. These investigations suggested that members of the Nitraria genus contain numerous components categorized as alkaloids, flavonoids and phenolic acid, of which alkaloids and flavonoids are the most abundant constituents. An overview of ingredients contained in the Nitraria genus is presented in Table II.

Numerous studies (5-36) have been conducted to explore the alkaloids contained in the members of the Nitraria 
Table I. Species of the Nitraria genus and the geographical distribution.

\begin{tabular}{lll}
\hline No. & \multicolumn{1}{c}{ Species } & Geographical distribution \\
\hline 1 & N. schoberi & China, Europe \\
2 & N. sibirica & China, Siberia \\
3 & N. senegalensis & Africa \\
4 & N. billardieri & Austraria \\
5 & N. retusa & Mediterranean coast \\
6 & N. sphaerocarpa & Northwest China \\
7 & N.roborowskii & Northwest China, Russia \\
8 & N. komarovii & Europe caspian coast \\
9 & N.tangutorum & Northwest China \\
10 & N.praevisa & Northwest China \\
11 & N.pamirica & China \\
12 & N. sinensis & China \\
13 & N.tridentate & Africa \\
\hline
\end{tabular}

genus. Nearly all known alkaloids (Table II, nos. 1-36) identified in plants of the Nitraria genus were initially isolated in these studies. These studies also observed that the content of alkaloids in the leaves is higher than the content in the roots, stems and seeds of Nitraria komarovii and Nitraria sibirica (37-38). There are also various types of alkaloids that have been isolated and purified in other studies. In the crude $\mathrm{MeOH}$ extracts of $N$. rhoberi, nazlinin was purified by Üstunes et al (39). In a proceeding study, tangutorine was isolated from the leaves of $N$. tangutorum (40). Three novel alkaloids, 3-epinitrarine, 1-epinitraramine and nitrarine (41) were identified in N. billardieri, which is a species that is mainly distributed in Australia. Phytochemical studies of the aerial sections of $N$. retusa reported the novel alkaloids 5,7-dihydroxy-3-deoxy vasicine and 7-hydroxy-3-deoxy-1-vasiciene (42) (Fig. 1). Nitraria was also reported by Saleh et al (43) to contain the flavonoids rutin, kaempferol and isorhamnetin. The flavonoids isolated in other studies are listed in Table II, nos. 67-87 (44-48) (Fig. 2).

Wang et al (49) determined fatty acids in $N$. tangutorum seed by supercritical carbon dioxide extraction and high-performance liquid chromatography/atmospheric pressure chemical ionization/mass spectrometry. Using gas chromatograph-mass spectrometry, numerous volatile substances were detected in the extract of the stem, leaves and fruits of $N$. tangutorum and $N$. sibirica (50-52). Additionally, $\mathrm{Wu}$ et al (53) isolated phenolic acid from the water extraction of $N$. tangutorum.

\section{Pharmacological effects}

Antifatigue activity. The antifatigue activity of polysaccharides from the fruits of N. tangutorum was assessed in mice using the forced swim test (FST). The results demonstrated that the FST-induced reductions in glucose, superoxide dismutase (SOD) and glutathione peroxidase (GPx), and the increases in creatine phosphokinase, lactic dehydrogenase, blood urea nitrogen, triglyceride (TG) and malondialde- hyde (MDA) levels, were inhibited by the polysaccharides from $N$. tangutorum. Additionally, at the same dosage, the extract of $N$. tangutorum is more potent than Hippophae rhamnoides and Lycium ruthenicum, which are traditionally used as medicinal foods with antifatigue and antioxidant potential in Tibet (54). Fruit extracts of $N$. tangutorum markedly prolonged the swimming time, climbing time and survival time in low temperature of mice compared with the control group in a study by Suo et al (55). The seed oil of $N$. tangutorum, when extracted by supercritical $\mathrm{CO}_{2}$, displayed similar effects as the fruit, and upregulated the contents of serum urea-nitrogen and hepatic glycogen, but downregulated the serum lactic acid contents, consequently improving the swimming and climbing time. This result has been repeated in other studies $(56,57)$.

Antitumor activity. Boubaker et al investigated the apoptotic potential of $N$. retusa ethyl acetate (EA) extract and isorhamnetin 3-O-rutinoside (I3-O-R) isolated from the ethyl acetate extract, in K562 human chronic myelogenous erythroleukemia cells. After $48 \mathrm{~h}$ incubation with $N$. retusa extract and I3-O-R, K562 cell viability was significantly suppressed by inducing apoptosis, and the caspase 3 and caspase 8 activity was increased (58). The extract and the component inhibited the genotoxicity induced by hydroxyl radicals in K562 cells (59). Another similar study indicated that EA extract of $N$. retusa and I3-O-R have a strong antiproliferative effect on TK6 human lymphoblastoid cells, possibly due to their involvement in the apoptotic pathway (60).

The hexane (Hex), chloroform $(\mathrm{Chl})$ and methanol $(\mathrm{MeOH})$ extracts of $N$. retusa were utilized to test their antiproliferative effects on K562 cells. The Hex and Chl extracts were demonstrated to induce stronger antiproliferative effects than the $\mathrm{MeOH}$ extracts, by ameliorating the DNA fragmentation, poly ADPribose polymerase cleavage, and caspase 3 and caspase 8 activity (61). In another study, 3H-thymidine incorporation-induced proliferation of the HT29 human colon cancer cells was reduced in a dose-dependent manner following treatment with tangutorine, a $\beta$-carboline alkaloid from the leaves of $N$. tangutorum. Tangutorine may induce p21 suppression of all cyclins and their associated kinases, such as the topoisomerase II, and thus inhibit normal DNA replication and mitosis (62). The activities of fractions/extracts of $N$. retusa were compared with their flavanoid contents, which consisted of the following four major flavonoids: Isorhamnetin; isorhamnetin-3-O-glucoside (I3-O-G); I3-O-R; and isorhamnetin-3-O-robinobioside (I3-O-Rb). They inhibited the proliferation of Caco-2 cells in vitro (63).

The total flavones from the $N$. tangutorum fruits repress proliferation of the SGC-7901 human gastric adenocarcinoma cell line and A-704 human kidney adenocarcinoma cell line by regulating the levels of $\mathrm{Ca}^{2+}, \mathrm{K}^{+}$and $\mathrm{P}^{3+}$ in the cell (64-65). The in vivo experiment demonstrated that flavones, in addition to the aqueous extract of $N$. tangutorum, combined with 5-fluorouracil, induced a significantly increased inhibitory rate in the Hep human throat cancer cell line and U14 human cervical cancer cell line by regulating the weight of immune organs, the formation value of serolysin and phagocytic index (66-67). Additionally, the lipids of N.tangutorum were cytotoxic against the MGC-803 human gastric carcinoma cell line (68). 
Table II. Compounds isolated from the Nitraria genus.

A, Alkaloid compounds

\begin{tabular}{|c|c|c|c|c|}
\hline No. & Compound & Species & Part of plant & Reference \\
\hline 1 & Nitraricine & N. komarovii & Epigeal & 5 \\
\hline 2 & Nitrarizine & N. komarovii & Epigeal & 5 \\
\hline 3 & Isokomarovine & N. komarovii & Epigeal & 6 \\
\hline 4 & Komarovidinine & N. komarovii & Epigeal & 6 \\
\hline 5 & Komarovinine & N. komarovii & Epigeal & 7 \\
\hline 6 & Peganine N-oxide & N. komarovii & Epigeal & 8 \\
\hline 7 & Nallylschoberine & N. komarovii & Epigeal & 8 \\
\hline 8 & Dehydronitramidine & N. komarovii & Epigeal & 8 \\
\hline 9 & Komavicine & N. komaroviii & Epigeal & 9 \\
\hline 10 & Deoxypeganine & N. komarovii & Epigeal & 10 \\
\hline 11 & Nitraramine & N. schoberi/N. komarovii & Epigeal & 11 \\
\hline 12 & Komarovine & N. komarovii & Epigeal & 12 \\
\hline 13 & Komarovidine & N. komarovii & Epigeal & 12 \\
\hline 14 & Komaroine & N. komarovii & Epigeal & 13 \\
\hline 15 & Nitraraine & N. schoberi & Epigeal & 14 \\
\hline 16 & Nitrabirine & N. sibirica & Epigeal & 14 \\
\hline 17 & Nitramine & N. sibirica & Epigeal & 15 \\
\hline 18 & Isonitramine & N. sibirica & Epigeal & 16 \\
\hline 19 & Sibirine & N. sibirica & Epigeal & 17 \\
\hline 20 & Vasicinone $\mathrm{N}$-oxide & N. komarovii & Epigeal & 18 \\
\hline 21 & Deoxyvasicinone $\mathrm{N}$-oxide & N. komarovii & Epigeal & 18 \\
\hline 22 & Dihydronitraraine & N. komarovii & Epigeal & 19 \\
\hline 23 & Deoxyvasicinone & N. komarovii & Epigeal & 20 \\
\hline 24 & Vasicinone & N. komarovii & Epigeal & 20 \\
\hline 25 & Peganine & N. komarovii & Epigeal & 20 \\
\hline 26 & Deoxypeganine & N. komarovii & Epigeal & 20 \\
\hline 27 & Dehydroschoberine & N. komarovii & Epigeal & 20 \\
\hline 28 & Nitraroxine & N. komarovii & Epigeal & 20 \\
\hline 29 & Tryptamine & N. komarovii & Epigeal & 20 \\
\hline 30 & Schoberine & N. komarovii & Epigeal & 20 \\
\hline 31 & Schoberidine & N. komarovii & Epigeal & 21 \\
\hline 32 & Nitrarine & N. schoberi & Epigeal & 22 \\
\hline 33 & $\begin{array}{l}\text { Tetramethylenetetrahydro- } \beta \text { - } \\
\text { carboline N-oxide }\end{array}$ & N. komarovii & Epigeal & 23 \\
\hline 34 & Sibirinine & N. sibirica & Epigeal & 24 \\
\hline 35 & Dihydroschoberine & N. sibirica & Aerial & 25 \\
\hline 36 & Nitrabirine N-oxide & $N$. sibirica & Aerial & 25 \\
\hline 37 & O-acetylnitraraine & N. schoberi & Aerial & 26 \\
\hline 38 & $\mathrm{~N}$-methylnitrarine & N. schoberi & Aerial & 27 \\
\hline 39 & Komavine & N. komarovii & Aerial & 28 \\
\hline 40 & Acetylkomavine & N. komarovii & Aerial & 28 \\
\hline 41 & N-allylnitrarine & N. komarovii & Aerial & 29 \\
\hline 42 & Komarovidinine $\mathrm{N}$-oxide & N. komarovii & Aerial & 29 \\
\hline 43 & Sibiridine & N. sibirica/N. schoberi & Aerial & 30 \\
\hline 44 & Nitraramidine & N. sibirica & Aerial & 31 \\
\hline 45 & Nitraraidine & N. sibirica & Aerial & 31 \\
\hline 46 & Komarin & N. komarovii & Aerial & 32 \\
\hline 47 & Peganol N-oxide & N. komarovii & Aerial & 32 \\
\hline 48 & $\mathrm{~N}$-allylisonitrarine & N. schoberi & Aerial & 33 \\
\hline 49 & Nitraridine & N. komarovii & Aerial & 34 \\
\hline
\end{tabular}


Table II. Continued.

\begin{tabular}{|c|c|c|c|c|}
\hline No. & Compound & Species & Part of plant & Reference \\
\hline 50 & Dihydronitraridine & N. komarovii & Aerial & 34 \\
\hline 51 & Tetrahydronitraridine & N. Komarovii & Aerial & 34 \\
\hline 52 & Schobericine & N. Schoberi & Aerial & 35 \\
\hline 53 & Komaroidine & N. Komarovii/N. Schoberi & Aerial & 35 \\
\hline 54 & Acetylkomaroidine & N. Komarovii/N. Schoberi & Aerial & 35 \\
\hline 55 & Tetrahydronitramarine & N. Komarovii. & Epigeal & 36 \\
\hline 56 & Tetrahydrokomarovinine & N. Komarovii & Epigeal & 36 \\
\hline 57 & Dihydroisokomarovine & N. Komarovii & Epigeal & 36 \\
\hline 58 & Tetrahydroisokomarovine & N. Komarovii & Epigeal & 36 \\
\hline 59 & Nazlinin & N. Schoberi & Epigeal & 39 \\
\hline 60 & Tangutorine & N. Tangutorum & Leaves & 40 \\
\hline 61 & Nitrarine & N. Billardieri & Aerial & 41 \\
\hline 62 & 1-Epinitraramine & N. Billardieri & Aerial & 41 \\
\hline 63 & 3-Epinitrarine & N. Billardieri & Aerial & 41 \\
\hline 64 & $\begin{array}{l}\text { 5,7-Dihydroxy-3-deoxy- } \\
\text { vasicine }\end{array}$ & N. Retusa & Aerial & 42 \\
\hline 65 & $\begin{array}{l}\text { 7-Hydroxy-3-deoxy-1- } \\
\text { vasiciene }\end{array}$ & N. Retusa & Aerial & 42 \\
\hline 66 & Allantoin & N. Tangutorum & Seed & 48 \\
\hline
\end{tabular}

B, Flavanoid compounds

\begin{tabular}{|c|c|c|c|c|}
\hline No. & Compound & Species & Part of plant & Reference \\
\hline 67 & Narcissin & N. Komarovii & Leaves & 28 \\
\hline 68 & Rutin & N. Retusa & Leaves and stems & 43 \\
\hline 69 & Kaempferol & N. Retusa & Leaves and stems & 43 \\
\hline 70 & $\begin{array}{l}\text { Isorhamnetin } 3-\mathrm{O}-4^{\text {rham_- }} \\
\text { galactosylrobinobioside }\end{array}$ & N. Retusa & Leaves and stems & 44 \\
\hline 71 & Isorhamnetin 3-robinobioside & N. Retusa & Leaves and stems & 44 \\
\hline 72 & Isorhamnetin 3-rutinoside & N. Retusa & Leaves and stems & 44 \\
\hline 73 & Isorhamnetin 3-galactoside & N. Retusa & Leaves and stems & 44 \\
\hline 74 & Isorhamnetin 3-glucoside & N. Retusa & Leaves and stems & 44 \\
\hline 75 & Isorhamnetin & N. Retusa & Leaves and stems & 44 \\
\hline 76 & $\begin{array}{l}\text { Isorhamnetin } 3 \text { - } \\
\text { xylosylrobinobioside }\end{array}$ & N. Retusa & Leaves and stems & 44 \\
\hline 77 & $\begin{array}{l}\text { Isorhamnetin-7-O- } \alpha-\mathrm{L}- \\
\text { rhamnoside }\end{array}$ & N. Tangutorum & Seeds & 45 \\
\hline 78 & $\begin{array}{l}\text { Isorhamnetin-7-O- } \beta-\mathrm{D}- \\
\text { glucoside }\end{array}$ & N. Tangutorum & Seeds & 45 \\
\hline 79 & $\begin{array}{l}\text { Kaempferol-7- } O-\alpha-\mathrm{L}- \\
\text { rhamnoside }\end{array}$ & N. Tangutorum & Seeds & 45 \\
\hline 80 & $\begin{array}{l}\text { Quercetin-7-O- } \alpha-\mathrm{L}- \\
\text { rhamnoside }\end{array}$ & N. Tangutorum & Seeds & 45 \\
\hline 81 & Quercetin & N. Tangutorum & Seeds & 45 \\
\hline 82 & $\begin{array}{l}\text { 3,5-Dimethylether-kaempfrol- } \\
7-O-\beta \text {-D-glucoside }\end{array}$ & N. Tangutorum & Leaf & 46 \\
\hline 83 & $\begin{array}{l}\text { 3-Methylether-kaempferol- } \\
\text { 7-O- } \beta \text {-D-glucoside }\end{array}$ & N. Tangutorum & Leaf & 46 \\
\hline 84 & $\begin{array}{l}\text { Isorhamnetin-3-O- } \beta \text {-D- } \\
\text { glucopyranosyl-(1-2)- } \alpha \\
\text {-L-rhamnopyranoside }\end{array}$ & N. Tangutorum & Fruit & 47 \\
\hline 85 & 5,7,2'-Trihydroxyflavonol & N. Tangutorum & Fruit & 47 \\
\hline
\end{tabular}


Table II. Continued.

\begin{tabular}{llll}
\hline No. & \multicolumn{1}{c}{ Compound } & Species & Part of plant \\
\hline 86 & $\begin{array}{l}\text { Cyaniding 3-[6'-(6-trans-p- } \\
\text { coumaroyl- } \beta \text {-D-glucopyranosyl)- } \\
\beta \text {-D-galactopyranoside] } \\
\text { Apigenin 5-O-(2'-O-E-P- } \\
\text { coumaroyl)- } \beta \text {-D-glucopyranoside }\end{array}$ & N. Tangutorum & Fruit \\
& N. Tangutorum & Fruit \\
\hline
\end{tabular}<smiles></smiles><smiles></smiles><smiles>c1cc2nccc3c2c(c1)c1nccc2c4ccccc4n3c21</smiles><smiles>c1cnc2ccc(-c3nccc4c3[nH]c3ccccc34)cc2c1</smiles>

3<smiles>NC/C=C\c1cccc(-c2nccc3c2[nH]c2ccccc23)c1</smiles>

9

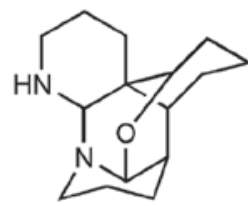

11

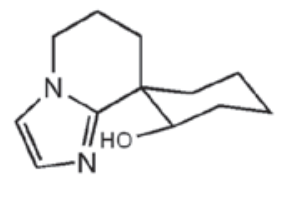

16<smiles>[R]C1CCn2c1[n+]([O-])c1ccccc1c2=O</smiles>

$20 \mathrm{R}=\mathrm{OH}$

$21 \mathrm{R}=\mathrm{H}$<smiles>C1=CN2C3C(C1)CCCC3CN1CCCCC12</smiles>

27<smiles>CC1OC2CCCCC23CCC[N+]1(O)C3</smiles>

34<smiles>c1cnc2c(-c3nccc4c3[nH]c3ccccc34)cccc2c1</smiles>

12<smiles>OC1CCCNC1</smiles>

17<smiles>OCC1CCCC2CN3CCc4c([nH]c5ccccc45)C3CC12</smiles>

22<smiles></smiles><smiles>C1CCN(CC2CCCC3CCCNC32)CC1</smiles>

35

Figure 1. Chemical structures of the main alkaloids from the genus Nitraria. 
<smiles>[R]NC12CCCCC1c1[nH]c3ccccc3c1CC2</smiles>

$39 \mathrm{R}=\mathrm{H}$

$40 \mathrm{R}=\mathrm{COCH}_{3}$<smiles></smiles>

46<smiles>CS(=O)(=O)c1ccc(C2NCCc3c2[nH]c2ccccc32)nc1</smiles>
56<smiles>C1=C(C2CCCCN2)c2c([nH]c3ccccc23)C2CCCCN12</smiles>

61

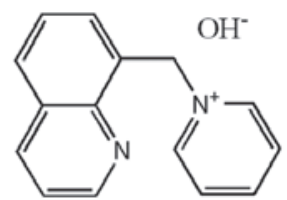

43<smiles>c1ccc2c(-c3nccc4c3[nH]c3ccccc34)ccnc2c1</smiles>

49<smiles>c1ccc2c(C3=NCCc4c3[nH]c3ccccc43)cccc2c1</smiles>

57<smiles>Oc1cc(O)c2c(c1)CN1CCCC1=N2</smiles>

64

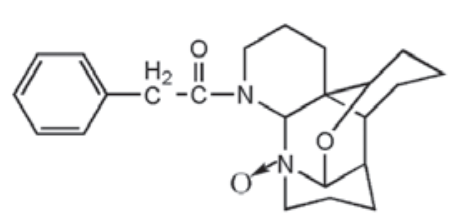

44<smiles></smiles>

$52 \mathrm{R}=\mathrm{OH} \quad \mathrm{R}_{1}=\mathrm{H}$

$53 \mathrm{R}=\mathrm{H} \quad \mathrm{R}_{1}=\mathrm{H}$

$54 \mathrm{R}=\mathrm{H} \quad \mathrm{R}_{1}=\mathrm{CH}_{3} \mathrm{CO}$<smiles>c1ccc2c3c([nH]c2c1)[C@H]1CC[C@H]2CC[C@@H](C1)CN2CC3</smiles>

45<smiles>c1ccc2nc(C3NCCc4c3[nH]c3ccccc43)ccc2c1</smiles><smiles>NC1CCCCNCCc2c1[nH]c1ccccc21</smiles>

59<smiles>Oc1ccc2c(c1)CN1C=CCC1=N2</smiles>

65<smiles>C[C@@]12CCC(CO)=C[C@H]1N1CCc3c([nH]c4ccccc34)[C@@]1(C)CC2</smiles>

60<smiles>NC(=O)NC1NC(=O)NC1=O</smiles>

66

Figure 1 continued. Chemical structure of main alkaloids from the genus Nitraria.

Antioxidant activity. EA extract of $N$. retusa and I3-O-R indicated a protective effect against lipid peroxidation induced by $\mathrm{H}_{2} \mathrm{O}_{2}$. It demonstrated significant antioxidant effects on oxidation induced by 2,2'-azobis (2-amidinopropane) dihydrochloride in K562 cells with 50\% inhibitory concentration values (IC50) of $0.225 \mathrm{mg} / \mathrm{ml}$ and $0.31 \mathrm{mg} / \mathrm{ml}$ (59). Sterols, the main constituents of Hex extract, and sterol and polyphenolic compounds, the main constituents of Chl extract, may participate in the protective effect against lipid peroxidation induced by $\mathrm{H}_{2} \mathrm{O}_{2}$ in $\mathrm{K} 562$ cells (61). Chl extract exhibited the highest ability to protect plasmid DNA against hydroxyl radical-induced DNA damage, and the highest antioxidant capacity, with $0.95 \mathrm{mM}$ trolox equivalent capacity when tested using the ferric reducing/antioxidant method (69). Furthermore, the extract of fruits from $N$. tangutorum displayed a significantly different antioxidant activity when assessed with the 1,1-diphenyl-2-picrylhydrazy (DPPH), 2,2'-Azinobis (3-ethylbenzothiaz-oline-6-sulfonate) (ABTS) and ferric-reducing antioxidant power assays (70).

The DPPH scavenging activity, xanthine oxidase inhibition and superoxide scavenging activity of various $N$. retusa extracts and compounds, including isorhamnetin, I3-O-G,
$\mathrm{I} 3-O-\mathrm{R}$ and $\mathrm{I} 3-O-\mathrm{Rb}$, were evaluated to confirm the association between the activities of the fractions and their flavonoid contents. The EA extracts were identified to be most effective at scavenging the DPPH stable free radical, and the $\mathrm{CHCl}_{3}$ extracts exhibited the highest xanthine oxidase inhibition activity; however, only $\mathrm{BuOH}$ extract exhibited a scavenging activity toward superoxide radicals. Overall, all the compounds exhibited some level of DPPH and superoxide scavenging and xanthine oxidase inhibition activity, and the aglycone compounds were more active than their glycosylated derivatives (63). Antiradical activities against DPPH, and $\beta$-carotene and Fe-reducing power were more efficient in leaf non-polar fractions compared with polar fractions of $N$. retusa (61). Similar studies investigating anti-oxidant properties were also performed by Bouaziz et al (71) and demonstrated that the EA fraction and $\mathrm{MeOH}$ fraction of $N$. retusa indicated DPPH scavenging activity and reduction of the ABTS radical cation.

Anthocyanins have also been demonstrated to elicit scavenging effects against $\mathrm{O}_{2}, \mathrm{OH}$ and DPPH in a dose-dependent manner. Notably, these scavenging capacities were greater than those of vitamin $\mathrm{C}$ according to results of in vitro anti-oxidative tests (72). Nazlinin isolated from Nitraria and 
<smiles>[R]c1cc([R2])c2c(=O)c(C([R])(C)O)c(-c3ccc([R6])c([R])c3[R])oc2c1</smiles>

$67 \mathrm{R}_{1}=\mathrm{OH} \mathrm{R} \quad \mathrm{R}_{2}=\mathrm{OH} \mathrm{R} \mathrm{R}_{3}=$ glu $\mathrm{R}_{4}=\mathrm{OH} \mathrm{R} \quad \mathrm{R}_{5}=\mathrm{OH} \mathrm{R} \mathrm{R}_{6}=\mathrm{H}$

$68 \mathrm{R}_{1}=\mathrm{H}_{2}=\mathrm{OH} \quad \mathrm{R}_{3}=$ glu $\quad \mathrm{R}_{4}=\mathrm{OH} \quad \mathrm{R}_{5}=\mathrm{OH} \quad \mathrm{R}_{6}=\mathrm{H}$

$69 \mathrm{R}_{1}=\mathrm{OCH}_{3} \quad \mathrm{R}_{2}=\mathrm{OH} \quad \mathrm{R}_{3}=$ bobinobioside $\quad \mathrm{R}_{4}=\mathrm{OH} \quad \mathrm{R}_{5}=\mathrm{OH} \quad \mathrm{R}_{6}=\mathrm{H}$

$71 \quad \mathrm{R}_{1}=\mathrm{OCH}_{3} \quad \mathrm{R}_{2}=\mathrm{OH} \quad \mathrm{R}_{3}=\beta$-D-rutinoside $\mathrm{R}_{4}=\mathrm{OH} \quad \mathrm{R}_{5}=\mathrm{OH} \quad \mathrm{R}_{6}=\mathrm{H}$

$72 \mathrm{R}_{1}=\mathrm{OCH}_{3} \quad \mathrm{R}_{2}=\mathrm{OH} \mathrm{R} \mathrm{R}_{3}=$ galactoside $\mathrm{R}_{4}=\mathrm{OH} \mathrm{R} \quad \mathrm{R}_{5}=\mathrm{OH} \mathrm{R} \mathrm{R}_{6}=\mathrm{H}$

$73 \quad \mathrm{R}_{1}=\mathrm{OCH}_{3} \quad \mathrm{R}_{2}=\mathrm{OH} \mathrm{R} \quad \mathrm{R}_{3}=$ glu $\quad \mathrm{R}_{4}=\mathrm{OH} \quad \mathrm{R}_{5}=\mathrm{OH} \quad \mathrm{R}_{6}=\mathrm{H}$

$74 \quad \mathrm{R}_{1}=\mathrm{OCH}_{3} \quad \mathrm{R}_{2}=\mathrm{OH} \mathrm{R} \mathrm{R}_{3}=\mathrm{H}_{4}=\mathrm{OH} \mathrm{R} \quad \mathrm{R}_{5}=\mathrm{OH} \mathrm{R} \mathrm{R}_{6}=\mathrm{H}$

$75 \quad \mathrm{R}_{1}=\mathrm{OCH}_{3} \quad \mathrm{R}_{2}=\mathrm{OH} \quad \mathrm{R}_{3}=x y$ losylrobinobioside $\mathrm{R}_{4}=\mathrm{OH} \quad \mathrm{R}_{5}=\mathrm{OH} \quad \mathrm{R}_{6}=\mathrm{H}$

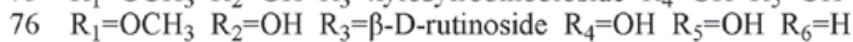

$77 \quad \mathrm{R}_{1}=\mathrm{OCH}_{3} \quad \mathrm{R}_{2}=\mathrm{O}-\alpha$-L-rhamnoside $\quad \mathrm{R}_{3}=\mathrm{H}_{4} \quad \mathrm{R}_{4}=\mathrm{OH} \quad \mathrm{R}_{5}=\mathrm{OH} \quad \mathrm{R}_{6}=\mathrm{H}$

$78 \quad \mathrm{R}_{1}=\mathrm{OCH}_{3} \quad \mathrm{R}_{2}=\mathrm{O}-\beta$-D-glu $\mathrm{R}_{3}=\mathrm{H} \mathrm{R}_{4}=\mathrm{OH} \quad \mathrm{R}_{5}=\mathrm{OH} \mathrm{R} \mathrm{R}_{6}=\mathrm{H}$

$79 \quad \mathrm{R}_{1}=\mathrm{H} \quad \mathrm{R}_{2}=\mathrm{O}-\alpha$-L-rhamnoside $\quad \mathrm{R}_{3}=\mathrm{H} \quad \mathrm{R}_{4}=\mathrm{OH} \quad \mathrm{R}_{5}=\mathrm{OH} \quad \mathrm{R}_{6}=\mathrm{H}$

$80 \mathrm{R}_{1}=\mathrm{OH} \mathrm{R} \quad \mathrm{R}_{2}=\mathrm{O}-\alpha$-L-rhamnoside $\mathrm{R}_{3}=\mathrm{H}_{4} \quad \mathrm{R}_{4}=\mathrm{OH} \quad \mathrm{R}_{5}=\mathrm{OH} \quad \mathrm{R}_{6}=\mathrm{H}$

$81 \quad \mathrm{R}_{1}=\mathrm{OH} \quad \mathrm{R}_{2}=\mathrm{OH} \quad \mathrm{R}_{3}=\mathrm{H}_{4}=\mathrm{OH} \quad \mathrm{R}_{5}=\mathrm{OH} \quad \mathrm{R}_{6}=\mathrm{H}$

$82 \mathrm{R}_{1}=\mathrm{H} \mathrm{R}_{2}=\mathrm{O}-\beta$-D-glu $\quad \mathrm{R}_{3}=\mathrm{CH}_{3} \quad \mathrm{R}_{4}=\mathrm{OCH}_{3} \quad \mathrm{R}_{5}=\mathrm{OH} \quad \mathrm{R}_{6}=\mathrm{H}$

$83 \quad \mathrm{R}_{1}=\mathrm{H} \quad \mathrm{R}_{2}=\mathrm{O}-\beta$-D-glu $\mathrm{R}_{3}=\mathrm{CH}_{3} \quad \mathrm{R}_{4}=\mathrm{OH} \quad \mathrm{R}_{5}=\mathrm{OH} \quad \mathrm{R}_{6}=\mathrm{H}$

$84 \mathrm{R}_{1}=\mathrm{OCH}_{3} \quad \mathrm{R}_{2}=\mathrm{OH} \mathrm{R}=\mathrm{rha}(2-1)$-glu $\mathrm{R}_{4}=\mathrm{OH} \mathrm{R} \quad \mathrm{R}_{5}=\mathrm{OH} \quad \mathrm{R}_{6}=\mathrm{H}$

$85 \mathrm{R}_{1}=\mathrm{H} \mathrm{R}_{2}=\mathrm{OH} \quad \mathrm{R}_{3}=\mathrm{H} \mathrm{R}_{4}=\mathrm{OH} \quad \mathrm{R}_{5}=\mathrm{H} \mathrm{R}_{6}=\mathrm{OH}$

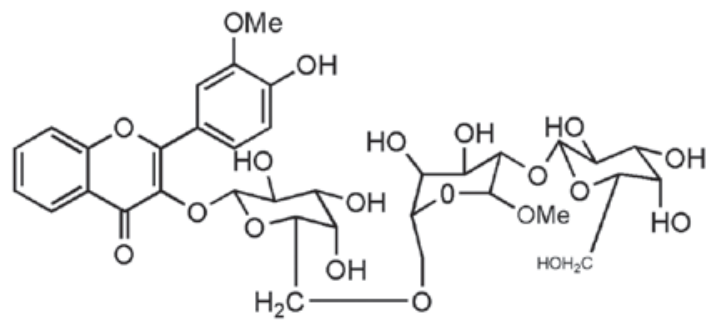

70

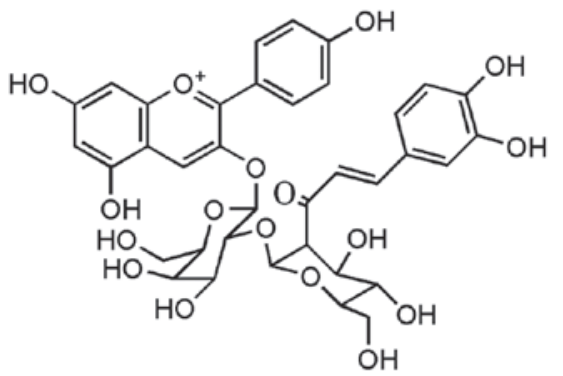

86<smiles></smiles>

87

Figure 2. Chemical structures of flavonoids from the genus Nitraria.

its derivative 1-(4-butylamino)-3,4-dihydro- $\beta$-carboline have been indicated to be inhibitors of pig kidney diamine oxidase, while 1-(4-butylamino)- $\beta$-carbolin was demonstrated as a substrate (73).

Antimicrobial activity. The EA, ethanol and Chl extracts from the fruits of $N$. tangutorum had antibacterial effects against
Escherichia coli, Bacillus subtilis and Staphyloccocus aureus. The EA fraction presented the highest level of antibacterial activity $(74,75)$. In addition, $\mathrm{Chl}$ extract from $N$. retusa leaves was more efficient against all human pathogen strains, particularly Escherichia coli and Staphylococcus aureus (76). The EA and $\mathrm{MeOH}$ extracts of $N$. retusa revealed antimicrobial effects against Pseudomonas aeruginosa and 
Aspergillus niger in vitro (71). The ethanol extracts of $N$. retusa also exhibited cytotoxicity in brine shrimp with LC50 values of $6.2 \mu \mathrm{g} / \mathrm{ml}(77)$.

Antimutagenic activity. The protection of $N$. retusa against mutagenicity induced by methyl methanesulfonate and 2-aminoanthracene in Salmonella typhimurium TA102 and TA104 strains was observed. The highest protection was elicited by $\mathrm{Chl}$ and $\mathrm{MeOH}$ extracts of $N$. retusa, with inhibition percentages of $44.93 \%$ at $50 \mu \mathrm{g} /$ plate in the presence of TA102 strain and $38 \%$ at $10 \mu \mathrm{g} /$ plate in the presence of TA104 strain. Hex and $\mathrm{Chl}$ extracts have been demonstrated to reduce the mutagenicity induced by 2 -aminoanthracene with $83.4 \%$ in TA104 and $65.3 \%$ in the TA102 strain (69).

Hypotensive effects. Senejouxa et al conducted a study on the vasorelaxant activity and underlying mechanisms of hydroalcoholic extract from the fruits of $N$. sibirica on thoracic aortic rings isolated from Wistar rats. The study revealed that the hydroalcoholic extract was more effective in the induction of vasodilation of phenylephrine- than high $\mathrm{KCl}$-pre-contracted aortic rings with respective $\mathrm{E}_{\max }$ values of $82.9 \pm 2.2$ and $34.8 \pm 3.6 \%$. The acute intravenous injection of hydroalcoholic extract induced an immediate and transient hypotensive effect in anesthetized spontaneously hypertensive and control rats through an endothelium-dependent pathway involving nitric oxide synthase (NOS) activation, endothelium-derived hyperpolarizing factor production and muscarinic receptor stimulation (78). Flavonoids of different concentrations increased the repair of impaired human umbilical vein endothelial cells induced by high glucose or $\mathrm{H}_{2} \mathrm{O}_{2}$ by increasing the ratio of NOS, SOD and GPx activity, and NO level $(79,80)$. Notably, the inhibition activity of ethanol extracts from 10 halophytes on angiotensin converting enzyme (ACE) has been investigated extensively. The Nitraria sibirica fraction has been demonstrated to significantly inhibit ACE with an IC50 value of $69.36 \mathrm{~g} / 1$ (81).

Hypoglycemic effects. The fruit of $N$. tangutorum at a dosage of $1.8 \mathrm{~g} / \mathrm{kg}$ and $3.6 \mathrm{~g} / \mathrm{kg}$ not only had a therapeutic action in a mouse diabetes model induced by alloxan, but also led to an increase in the glucose-toleration in similar models in rats. It has also been demonstrated to reduce glucose levels in hyperglycemic animal models induced by epinephrine and glucose (82). Shabana et al (83) investigated the hypoglycemic activity of 31 desert plants from different Egyptian localities in normal fasting and alloxanised rats, and Nitraria retusa had hypoglycemic effects in normal fasting rats.

Lipid lowering effects. An in vivo study on the effects of the fruit extracts of $N$. tangutorum on rat and mouse models of hyperlipermia induced by high lipid levels was conducted by Suo et al. N. tangutorum significantly reduced the serum level of total cholesterol (TC) and TG in a rat hyperlipermia model, and the level of low-density lipoprotein (LDL) in Kunming strain rats. It also increased the ratio of high-density lipoprotein cholesterol (HDL)/TC, HDL/LDL and SOD activity, and reduced the MDA content in vivo and in vitro $(84,85)$.

Hepatoprotective effects. A study by Zhang et al (86) indicated that seed oil of $N$. tangutorum alleviated the increased levels of aspartate aminotransferase, alanine aminotransferase and MDA induced by $\mathrm{CCl} 4$, and it also enhanced the level of GPx in liver.

\section{Conclusion}

The studies summarized above strongly support the theory that the Nitraria genus has favorable therapeutic properties, indicating its potential for clinical use. The present review presents and assesses the previous pharmacological and phytochemical studies published on the Nitraria genus, and may aid the easy identification and further research into properties of members of the Nitraria genus.

Nitraria as a halophyte, is ecologically central in stabilizing wind-blown sand and loess soils and thus reduces erosion. Hence, combined with the pharmacological effects, the rational development and utilization of Nitraria may be beneficial for the local environment and public health.

Numerous alkaloids have been isolated from the Nitraria genus. However, there is currently no research on the pharmacological properties of the alkaloid components, which are the most abundant constituents in nature. Further studies on the antitumor and anti-oxidative activities of these components are required. Furthermore, few molecular mechanisms are known, which may hamper the further clinical application of Nitraria. The possible synergistic action among the bioactive compounds of the plants must be evaluated prior to their use in clinical practice.

\section{Acknowledgements}

The current study was supported by grants from the National Natural Science Foundation of China (grant no. U1203104).

\section{References}

1. Liu YX: Desert Flora in China. Vol 2. Science Press, Beijing China, p306, 1988.

2. Wu Zhengyi, Raven H and Deyuan H: Flora of China. Vol. 43 (1) Science Press, Beijing, China, pp120-121, 1988.

3. Tackholm, V: Students Flora of Egypt, Cairo University: 31,313, 868,1974

4. Jafri S and El-Gadi A: Flora of Libya, Department of Botany, Al Fateh University: 55, 1977.

5. Tulyaganov TS and Abdullaev ND: Alkaloids of Nitraria komarovii Nitraricine and Nitrarizine. Chem Nat Compd 3: 76-78, 1995.

6. Tulyaganov TS, Ibragimov AA and Yunusov SY: Alkaloids of Nitraria komarovii. VI. structure and synthesis of isokomarovine and of komarovidinine, Chem Nat Compd 18: 601-603, 1982.

7. Tulyaganov TS, Ibragimov AA and Yunusov SY: Alkaloids of Nitraria komarovii. Vll. Structure and synthesis of komarovinine. Chem Nat Compd 18: 604-606, 1982.

8. Tulyaganov TS: Alkaloids of Nitraria komarovii XVII. Peganine $\mathrm{N}$-oxide, $\mathrm{N}$-allylschoberine and dehydronitramidine. Chem Nat Compd 30: 727-729, 1994.

9. Tulyaganov TS: Alkaloids of Nitraria komarovii. XI. Structure of komavicine and the products of the dehydrogenation of the nitrarine method of dehydrogenation in the quinolinyl- $\beta$-carboline series. Chem Nat Compd 29: 26-30, 1993.

10. Tulyaganov TS: Alkaloids of Nitraria komarovii. XIV. Deoxypeganine N-oxide. Chem Nat Compd 29: 73, 1993.

11. Ibragimov AA and Yunusov SY: Alkaloids of Nitraria schoberi. Ring-chain tautomerism of the hydrolysis product of nitraramine. Chem Nat Compd 22: 680-684, 1986.

12. Tulyaganov TS, Ibragimov AA and Yunusov SY: Alkaloids of Nitraria komarovii. IV. Total synthesis of komarovine and komarovidine. Chem Nat Compd 17: 149-152, 1981. 
13. Tulyaganov TS, Ibragimov AA and Yunusov SY: Alkaloids of Nitraria komarovii. IX. Structure of komaroine. Chem Nat Compd 20: 378-379, 1984.

14. Ibragimov AA and Yunusov SY: Alkaloids of Nitraria schoberi. Structure of nitraraine. Chem Nat Compd 21: 502-509, 1985.

15. Novgorodova NY, Maekh SK and Yunusov SY: Nitramine-A new alkaloid from Nitraria schoberi. Chem Nat Compd 11: 455-456, 1975.

16. Osmanov Z, Ibragimov AA and Yunusov SY: Isonitramine-A new alkaloid from Nitraria sibirica. Chem Nat Compd 13: 607-608, 1977.

17. Osmanov Z, Ibragimov AA and Yunusov SY: Structure of Sibirine. Chem Nat Compd 18: 206-208, 1982.

18. Tulyaganov TS, Atadzhanov RS, Abdullaev ND, et al: Alkloids of Nitraria komarovii. XV. Vasicinone and deoxyvasicinone N-oxides. Chem Nat Compd 29: 509-511, 1993.

19. Ibragimov AA and Yunusov SY: Alkaloids of Nitraria schoberi. Structure of dihydronitraraine. Chem Nat Compd 21: 510-512, 1985.

20. Tulyaganov TS: Alkaloids of Nitraria komarovii. XIII. Nitraramine n-oxide and the structure of dehydroschoberine. Chem Nat Compd 29: 31-34, 1993.

21. Ibragimov AA, Maekh SK and Yunusov SY: The structure of schoberine. Chem Nat Compd 11: 297, 1975.

22. Normatov M and Yunusov SY: A study of the alakloids of Nitraria Schoberi. The structure of nitrarine. Chem Nat Compd 4: 120, 1968.

23. Tulyaganov TS and Shorakhimov NN: Alkaloids of Nitraria komarovii XII. Tetramethylenetetrahydro- $\beta$-carboline N-oxide. Chem Nat Compd 26: 478-479, 1990.

24. Ibragimov AA, Abdullaev ND, Osmanov Z, et al: Alkaloids of Nitraria sibirica. Structure and absolute configuration of sibirinine. Chem Nat Compd 23: 569-573, 1987.

25. Tulyaganov TS and Alaberdiev FK: Alkaloids of Nitraria sibirica Dihydroschoberine and nitrabirine $\mathrm{N}$-oxide. Chem $\mathrm{Na}$ Compd 37: 556-557, 2007.

26. Tulyaganov TS and Kozimova NM: Alkaloids from Nitraria schoberi. O-acetylnitraraine. Chem Nat Compd 41: 578-579, 2005

27. Tulyaganov TS and Nazarov OM: Alkaloids of Nitraria schoberi. N-methylnitrarine. Chem Nat Compd 36: 393-394, 2000.

28. Tulyaganov TS, Nazarov OM, Levkovich MG, et al: Alkaloids of the Nitraria genus. Komavine and acetylkomavine. Chem Nat Compd 37: 61-64, 2001

29. Tulyaganov TS and Makhmudov OE: Alkaloids of Nitraria komarovii. N-allylnintrarine and komarovidinine N-oxide. Chem Nat Compd 36: 396-398, 2000.

30. Tulyaganov TS and Allaberdiev FK: Alkaloids from plants of the Nitraria genus. Structure of sibiridine. Chem Nat Compd 39: 292-293, 2003

31. Tulyaganov TS and Allaberdiev FK: Alkaloids of Nitraria sibirica. Structures of nitraramidine and nitraraidine. Chem Nat Compd 38: 602-604, 2002.

32. Tulyaganov TS and Makhmudov OE: Alkaloids of Nitraria komarovii. Structures of komarin and peganol-N-oxide. Chem Nat Compd 36: 76-78, 2000.

33. Tulyaganov TS, Nazarov OM, Makhmudov OE, et al: $\mathrm{N}$-allylisonitratine and narcissin from plants of the Nitraria genus. Chem Nat Compd 37: 470-473, 2001.

34. Tulyaganov TS: Alkaloids from Nitraria komarovii. Structures of nitraridine, dihydronitraridine and tetrahydronitraridine. Chem Nat Compd 42: 459-461, 2006.

35. Tulyaganov TS, Kozimova NM and Allaberdiev FK: Alkaloids from plants of the genus Nitraria. Chem Nat Compd 42: 198-200, 2006.

36. Tulyaganov TS and Yunusov SY: Alkaloids of Nitraria komarovii. $X$. Structures of tetrahydronitramarine, tetrahydrokomarovinine, dihydroisokomarovine, and tetrahydroisokomarovine. Chem Nat Compd 26: 49-53, 1990 .

37. Tulyaganov TS, Ibragimov AA and Yunusov SY: Alkaloids of Nitraria komarovii. I. Chem Nat Compd 15: 654, 1979.

38. Osmanov Z, Ibragimov AA, Yunusov SY, et al: Dynamics of the accumulation of the alkaloids of Nitraria sibirica. Chem Nat Compd 18: 372, 1982

39. Üstunes L, Özer A, Laekeman GM, et al: Chemical characterization and pharmacological activity of nazlinin, a novel indole alkaloid from Nitraria schoberi. J Nat Prod 54: 959-966, 1991.

40. Duan JA, Williams ID, Che CT, et al: Tangutorine: A nove $\beta$-carboline alkaloid from Nitraria tangutorum. Tetrahedron Lett 40: 2593-2596, 1999.
41. Shen MY, Zuanazzi JA, Kan C, et al: Alkaloids from Nitraria billardieri. Nat Prod Lett 6: 119-125, 1995.

42. El-Alali A, Zoubi AA, Gharaibeh M, et al: Phytochemical and biological investigation of Nitraria retusa. Asch. Jor J Pharm Sci 5: 155-163, 2012

43. Saleh, NAM and El-Hadidi MH: An approach to the chemosystematics of the Zygophyllaceae. Biochem Syst Ecol 5: 121-128, 1977.

44. Halim AF, Saad HE and Hashish NE: Flavonol glycosides from Nitraria retusa. Phytochemistry, 40: 349-351, 1995.

45. Jia ZJ, Zhu GJ and Wang JH: Flavonoid constituents from the seeds of Nitraria tangutorum Bolor. Acta Botanica Sinica 31: 241-243, 1989.

46. Duan JA, Zhou RH and Zhao SX: The chemical constituents of flavonoids and phenolic acid compounds of leaves from Nitraria tangutorum Bor. J Plant Resour Environ 8: 6-9, 1999.

47. Lu HL, Ouyang F, Su YL et al: Study on chemical constituents of the fruits of Nitraria tangutorum Bobr. Northwest Pharm J 28 221-223, 2013.

48. Wang HL, Li YL, Wang XY, et al: Chemical constituents of Nitraira tangutorum seed from Qaidam basin. Nat Prod Res Dev 19: 614-616, 2007.

49. Wang HL, Suo YR, Wang XY, et al: Extraction of Nitraria tangutorum seed oil by supercritical carbon dioxide and determination of free fatty acids by HPLC/APCI/MS with fluorescence detection. Sep Purif Technol 56:371-377, 2007.

50. Wang JM and Kang WY: Volatiles from the leaves of Nitraria tangutorum and Nitraria sibirica. Nat Prod Res Dev 23. 680-683, 2011

51. Wang JM and Kang WY: The volatiles in the stem of Nitraria tangutorum and Nitraria sibirica. Fine Chem 26: 773-775, 2009 .

52. Zhu Y, Liu JR, Wang HY, et al: Comparison and analysis on fruit oils of Nitraria prepared by supercritical $\mathrm{CO}_{2}$, fluid extraction and impregnation method. Food Fermentation Ind 32: 84-86, 2006.

53. Wu ZB, Li ML, Hang M, et al: Study on water-soluble chemical constituents of Nitraria tangutorum Bobr. J Shihezi Univ (Nat sci) 30: 728-730, 2012

54. Ni W, Gao T, Wang H, et al: Anti-fatigue activity of polysaccharides from the fruits of four Tibetan plateau indigenous medicinal plants. J Ethnopharmacol 150: 529-535, 2013.

55. Suo YR, Li YL, Wang HL, et al: Effect of fruit of Nitraria tangutorum Bobr. from Qaidam basin on immunomodulatory, anti-fatigue and cold-tolerance. Nat Prod Res Dev 17: 717-721, 2005.

56. Suo YR, Wang HL, Li YL, et al: The anti-fatigue effect of Nitraria tangutorum Bobr. Seed oil from Qaidam basin on the mice. Nat Prod Res Dev 18: 88-91, 2006

57. Ma TJ and Xu YQ: The anti-fatigue effect of Nitraria seed oil on mice. Acta Lab Anim Sci Sin 20: 77-79, 2012.

58. Boubaker J, Bhouri W, Sghaier MB, et al: Ethyl acetate extract and its major constituent, isorhamnetin 3-O-rutinoside, from Nitraria retusa leaves, promote apoptosis of human myelogenous erythroleukaemia cells. Cell Prolif 44: 453-461, 2011.

59. Boubaker J, Ben Sghaier M, Skandrani I, et al: Isorhamnetin 3-O-robinobioside from Nitraria retusa leaves enhance antioxidant and antigenotoxic activity in human chronic myelogenous leukemia cell line K562. BMC Complem Altern Med 12: 135, 2012.

60. Boubaker J, Bhouri W, Ben Sghaier M, et al: Flavonoids products from Nitraria retusa leaves promote lymphoblastoid cells apoptosis. Nutr Cancer 64: 1095-1102, 2012

61. Boubaker J, Bhouri W, Sghaier MB, et al: Leaf extracts from Nitraria retusa promote cell population growth of human cancer cells by inducing apoptosis. Cancer Cell Int 11: 37, 2011.

62. Liu BPL, Chong EYY, Cheung FWK et al: Tangutorine induces p21 expression and abnormal mitosis in human colon cancer HT-29 cells. Biochem Pharmacol 70: 287-299, 2005.

63. Salem JH, Chevalot I, Harscoat-Schiavo C, et al: Biological activities of flavonoids from Nitraria retusa (Forssk.) Asch. and their acylated derivatives. Food Chem 124: 486-494, 2011.

64. Liu CS, Wu XG, Xie MR, et al: Inhibition effects of Nitraria tangutorum flavone on SGC-7901 cell proliferation. J Gansu Sci 21: 66-68, 2009.

65. Yu FR, Lian SJ and Lian XZ: Cytotoxic and proliferous effects of Nitraria tangutorum flavone on human A-704 strain cells. Chin Pharmacol Bull 24: 236-239, 2008.

66. Yu FR, Wei KQ and Lian XZ: Effect and mechanism of nitrary flavone on Hep, U14 tumor of mice. Chin Pharmacol Bull 19: $886-888,2003$ 
67. Wei KQ, Yu FR and Lian XZ: Effect of nitrary decotion on rehabilitation of Hep tumor and reinforcement of immunological function. J Clin Rehab Tissue Eng 7: 2418-2419, 2003.

68. Li YP, Sun LP, Wang SZ, et al: Inhibition effects of shayingtao lipids on the human gastric carcinoma MGC-803 cells. Chin J Clin Res 26: 108-109, 2013

69. Boubaker J, Skandrani I, Bouhlel I, et al: Mutagenic, antimutagenic and antioxidant potency of leaf extracts from Nitraria retusa. Food Chem Toxicol 48: 2283-2290, 2010.

70. Zheng J, Li H, Ding CX, et al: Anthocyanins composition and antioxidant activity of two major wild Nitraria tangutorun Bobr. variations from Qinghai-Tibet Plateau. Food Res Int 44: 2041-2046, 2011.

71. Bouaziz M, Dhouib A, Loukil S, et al: Polyphenols content, antioxidant and antimicrobial activities of extracts of some wild plants collected from the south of Tunisia. Afric J Biotechn 8: 7017-7027, 2009.

72. Bai XM, Ding CX, Guo YQ, et al: Free radical scavenging effects of anthocyanins from the fruits of Nitraria tangutorun. Nat Prod Res Dev 20: 884-887, 2008.

73. Cheng E, Dekker HL, van Gelder BF, et al: Inhibition of pig kidney diamine oxidase by nazlinin and nazlinin derivatives Biochim Biophys Acta 1253: 189-192, 1995.

74. Zhang GX, Qi JH, Ren X, et al: In vitro antimicrobial activity of extracts from fruits of Nitraria sibirica pall. Sci Tech Food Ind 13: 104-106, 2012.

75. Li J, Wu XL, Chen Y, et al: Antidiarrheal properties of different extracts of Chinese herbal medicine formula Bao-Xie-Ning. J Integr Med 11: 125-134, 2013.

76. Zaouali Y, Ksouri WM, Saada M, et al: Solvent effects on antioxidants and biological activities of the halophyte Nitraria retusa (Forssk.) Asch Planta Med 77: PM203, 2011.

77. Tawaha KA: Cytotoxicity evaluation of Jordanian wild plants using brine shrimp lethality test. Jor J App Sci-Nat Sci 8: 12-17, 2006.
78. Senejouxa F, Girarda C, Aisab HA, et al: Vasorelaxant and hypotensive effects of a hydroalcoholic extract from the fruits of Nitraria sibirica Pall. (Nitrariaceae). J Ethnopharmacol 141: 629-634, 2012.

79. Fan LL, Tang H, Zhang YJ, et al: The protection of total flavonoids of Nitraria tangutorun Bobr. on vascular endothelial cells which was injured by high glucose. Pharm Clin Chin Mater Med 26: 21-23, 2010 (In Chinese)

80. Suo YR, Gao H and Wang HL: The protective effect of Nitraria tangutorum Bobr. seed oil from Qaidam basin on the liver injury of mice. Nat Prod Res Dev 17: 573-576, 2005.

81. Liu Y, Tan CY, Wu D, et al: Study on inhibition activity of angiotensin I -converting enzyme (ACE) on 10 halophytes. Chin Pharm J 45: 736-738, 2010 (In Chinese)

82. Suo YR and Wang HQ: Studies on hypoglycemic effect of fruit of Nitraria tangutorum Bobr. from Qinghai Tsaidam basin. Food Sci 25: 164-167, 2004

83. Shabana MM, Mirhom YW, Genenah AA, et al: Study into wild Egyptian plants of potential medicinal activity. Ninth communication: hypoglycaemic activity of some selected plants in normal fasting and alloxanised rats. Arch Exp Veterinarmed 44: 389-394, 1990.

84. Suo YR, Wang HL and Wang HQ: Research on decreasing blood lipid and anti-oxidative effect of fruit of Nitraria Tangutorum Bobr. From Qaidam basin. Nat Prod Res Deve 16: 54-58, 2004

85. Song HY, Zhang L, Pan JL, et al: Bioactivity of five components of Chinese herbal formula Jiangzhi granules against hepatocellular steatosis. J Integr Med 11: 262-268, 2013.

86. Zhang YJ, Fan LL, Zhang YJ, et al: Protective effect of total flavonoids of Nitraria tangutorun Bobr. against vascular endothelial cells injury induced by hydrogen peroxide. Lishizhen Med Materia Med Res 20: 562-564, 2009 (In Chinese). 